\title{
Effects of Increasing Electrodes on CNTs Yield Synthesized by Using Arc-Discharge Technique
}

\author{
Samy Yousef, ${ }^{1}$ A. Khattab, ${ }^{2}$ T. A. Osman, ${ }^{2}$ and M. Zaki ${ }^{1}$ \\ ${ }^{1}$ Department of Production Engineering and Printing Technology, Akhbar Elyom Academy, Giza 12566, Egypt \\ ${ }^{2}$ Department of Mechanical Design and Production Engineering, Cairo University, Giza 12566, Egypt \\ Correspondence should be addressed to Samy Yousef; ahcann@hotmail.com
}

Received 14 January 2013; Revised 25 April 2013; Accepted 7 May 2013

Academic Editor: Nadya Mason

Copyright (c) 2013 Samy Yousef et al. This is an open access article distributed under the Creative Commons Attribution License, which permits unrestricted use, distribution, and reproduction in any medium, provided the original work is properly cited.

\begin{abstract}
A new design of fully automatic system was built up to produce multiwalled carbon nanotube (MWCNT) using arc discharge technique in deionized water and extra pure graphite multiple electrodes (99.9\% pure). The goal of the experimental research is to determine the yield of CNT in two different cases: (a) single plasma electrodes and (b) multiplasma electrodes, particularly 10 electrodes. The experiments were performed at constant parameters (75 A, $238 \mathrm{~V})$. The obtained CNT was examined by scanning electron microscope (SEM), transmission electron microscope (HRTEM), X-ray diffraction (XRD), and thermogravimetric analysis (TGA). The results showed that the produced CNT is of type MWCNT, with a diameter of $5 \mathrm{~nm}$, when using multiplasma electrodes and $13 \mathrm{~nm}$ when using single plasma electrodes. The yield of MWCNT was found to be $320 \%$ higher in case of comparing multielectrodes to that of single plasma electrodes. Under the experimental test conditions, a yield of $0.6 \mathrm{~g} / \mathrm{hr}$ soot containing $40 \%$ by mass nanotube was obtained in case of single plasma electrodes and above $60 \%$ in case of multiplasma electrodes.
\end{abstract}

\section{Introduction}

Carbon nanotubes (CNTs) were observed in 1991 by Iijima who employed a method used to fabricate C60 fullerenes [1]. The properties and characteristics of CNTs are still being researched heavily and scientists have barely begun to tap the potential of these structures. CNTs have many unique and remarkable properties (chemical, physical, electrical, mechanical, and biomedical), which make them desirable for many applications, such as electronics, biology, medicine, energy, materials engineering, and aero science $[2,3]$. Therefore, CNT yield production is very important because it controls the price of the product. Rajashree et al. (2009) and Chai et al. (2004) reviewed in detail CNT technology and its applications [4-6]. The common different methods for synthesis CNT are arc discharge [7-17], laser vaporization [18], and chemical vapor deposition (CVD) [19-21]. Among several methods for preparing CNT, the arc discharge is the most practical method for scientific purposes because it yields highly graphitized tubes due to the high process temperature [22]. Multiwalled carbon nanotube (MWCNT) produced by electric arc discharge method is highly crystalline and exhibits fewer defects than MWNT produced by other methods $[23,24]$. In the arc discharge method, a voltage is applied across two graphite rods as electrodes. Carbon from the anode vaporizes and condenses on the cathode as nanotube, amongst other forms of carbon [25]. To increase the yield and the purity of CNT by arc discharge, many research works have been devoted to study the rate of yielding through the investigation of various effective parameters such as submerged media, current intensity, applied voltage, electrode size, and electrode movement speed [26-32]. After production of the nanoparticles, a purification method by sonication and centrifugal separation will be conducted. In the present research, a number of electrodes are used in a fully automatic system in order to produce MWCNT, using arc discharge technique in deionized water. The results are compared in terms of the yield and purification of the CNT, in addition to the thermal resistance with that of conventional single electrode. 


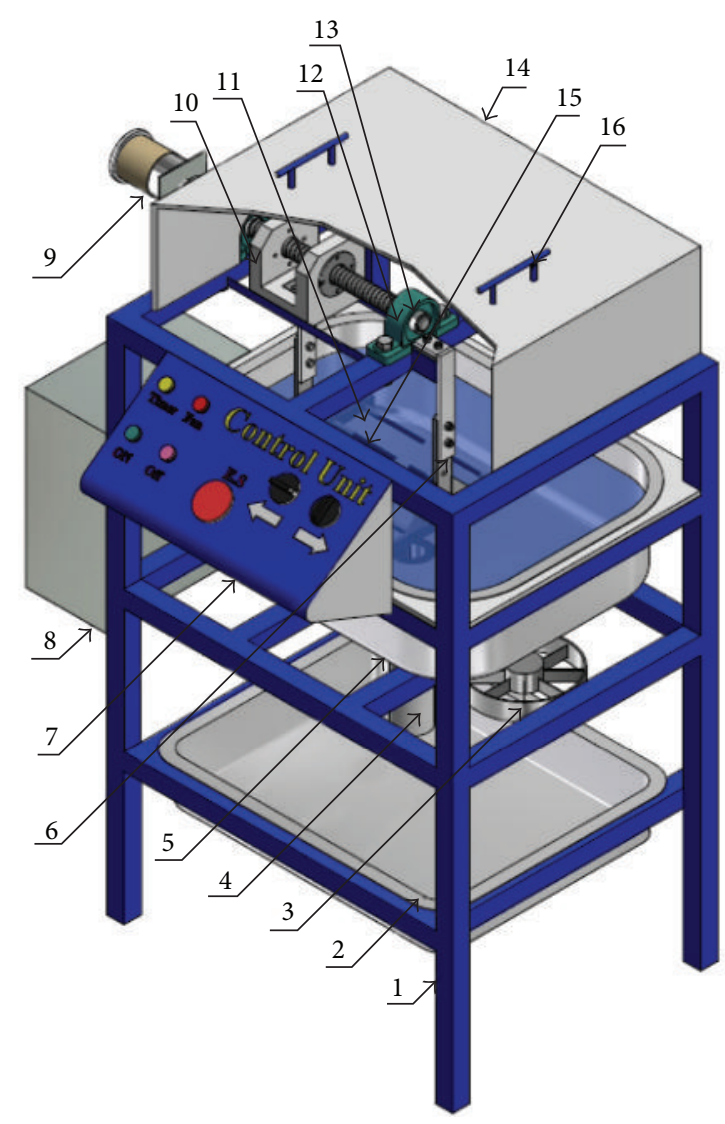

FIGURE 1: View of the fully automatic machine for producing CNTs. (1) Base frame, (2) lower tank, (3) fans for cooling, (4) valve, (5) upper tank, (6) carbon holder, (7) control unit, (8) electrical unit, (9) steeper motor, (10) nuts housing, (11) deionized water, (12) power screw, (13) bearing, (14) cover, (15) pure graphite, (16) cover hand.

\section{Experimental}

MWCNT was synthesized by a fully automatic designed apparatus using arc discharge technique in deionized water without catalyst at two different cases a single electrode and ten electrodes. The advantages of this apparatus compared to the existing arc discharge method are that it represents a cheap method equipped with fully automatic system. The gap between the electrodes can be set automatically by using a timer which results in an increase yield of carbon nanotubes. Figure 1 illustrates a view of the designed and manufactured machine. Five electrodes are clamped with left movable holder as cathodes which are moved by used power screw connected with steeper motor through an oldham coupling. Other five electrodes are clamped with the right fixed holder as anodes, as shown in Figure 2. A timer giving a signal to the steeper motor after every 70 seconds to compensate the electrodes consumption, the interval (70 seconds), has been identified experimentally. The height of the two holders can be adjusted by two bolts; Yousef et al. (2012) reviewed with details the designed system and its experimental procedure [33].

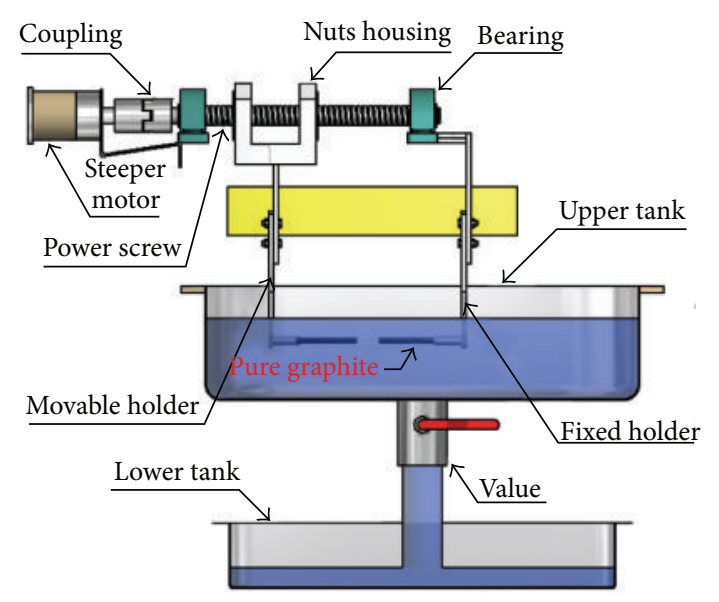

(a) Front view

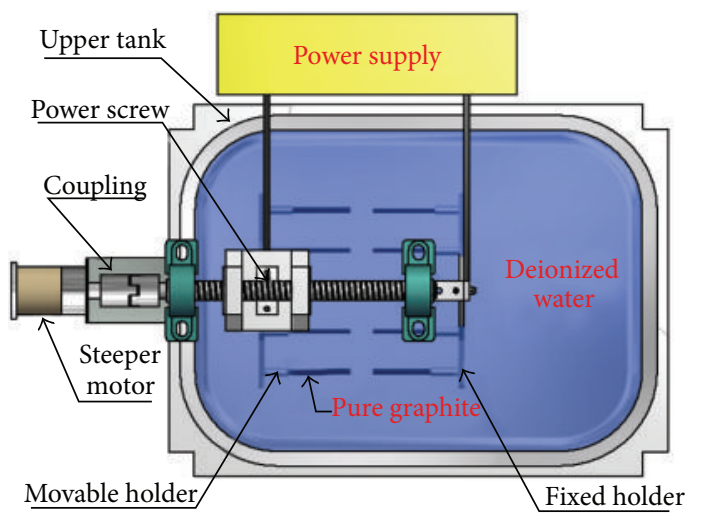

(b) Top view

FIgURE 2: Clamping the two electrodes in holders and phases of the bright plasma formation; (a) clamping the two electrodes, (b) gap sett, (c) initial plasma, and (d) formed fully bright plasma.

TABLE 1: Experimental conditions for single plasma electrodes case.

\begin{tabular}{ll}
\hline Parameters & Single plasma electrodes \\
\hline $\begin{array}{l}\text { Number of } \\
\text { cathodes }\end{array}$ & One graphite electrodes with $\varnothing 6 \mathrm{~mm}$ \\
$\begin{array}{l}\text { Number of anodes } \\
\text { Applied voltage }\end{array}$ & $\begin{array}{l}\text { One graphite electrodes with } \varnothing 6 \mathrm{~mm} \\
\text { Electric current }\end{array}$ \\
$\begin{array}{l}75 \mathrm{~A} \text { (AC arc-discharge) } \\
\text { Environment }\end{array}$ & \begin{tabular}{l} 
teoling air, deionized water, and room \\
\hline
\end{tabular}
\end{tabular}

2.1. Synthesizing MWCNT Using Single Plasma Electrodes. Two electrodes are used in this experiment. The two pure graphite electrodes are clamped to the fixed and movable holders presenting one electrode as a cathode and another as anode, as shown in Figure 3(a). The experiment has been performed under AC arc discharge, $75 \mathrm{~A}$ and $238 \mathrm{~V}$. The arc discharge and the resulted plasma are shown in Figures $3(\mathrm{~b})-3(\mathrm{~d})$, respectively. Table 1 summarizes the processing parameters used in this research work. 


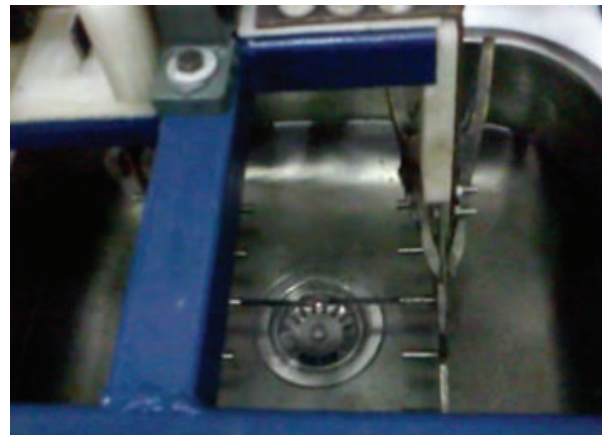

(a)

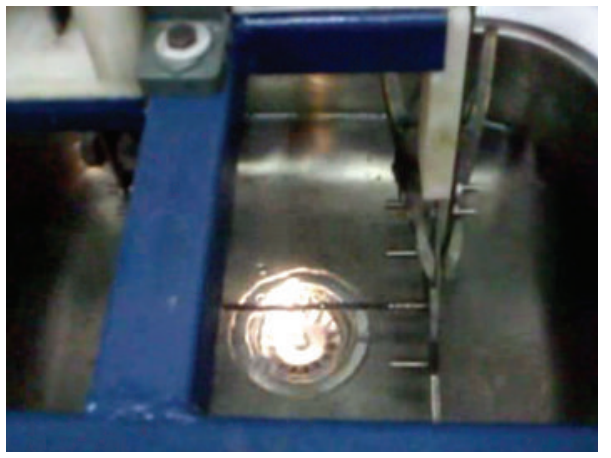

(c)

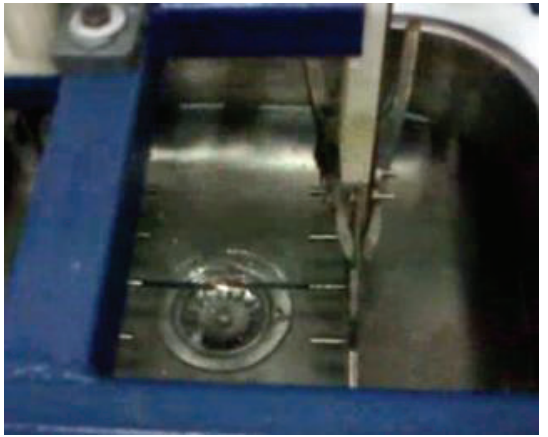

(b)

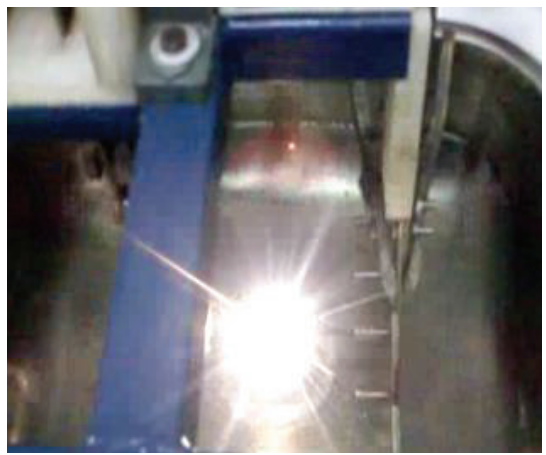

(d)

FIGURE 3: Clamping the two electrodes in holders and phases of the bright plasma formation.

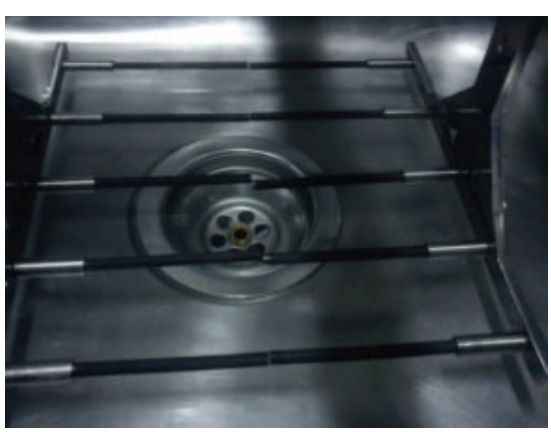

(a)

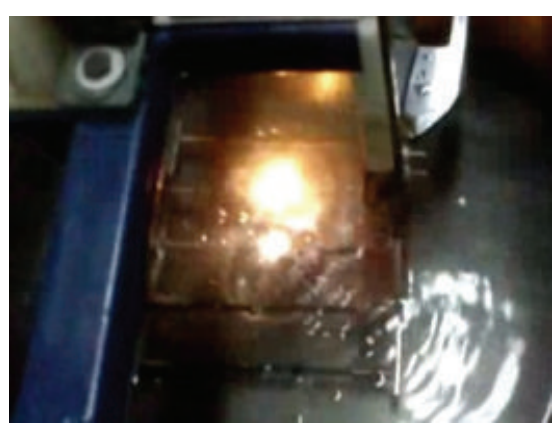

(d)

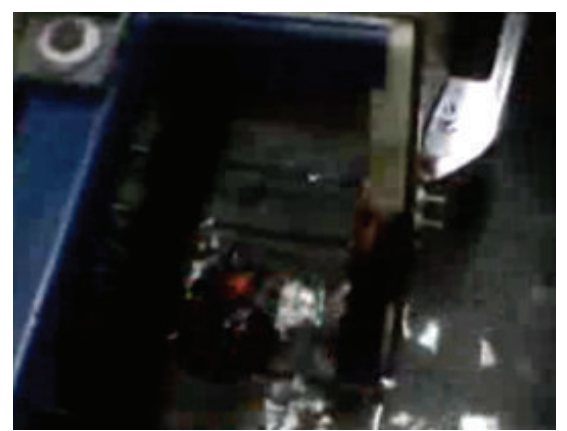

(b)

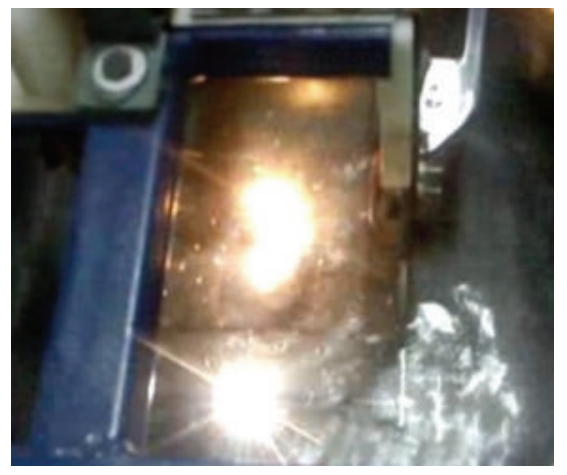

(e)

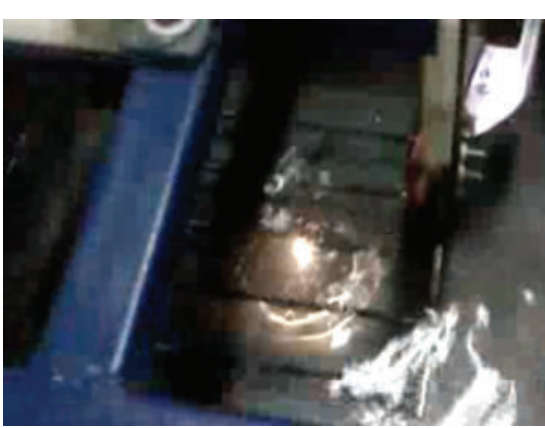

(c)

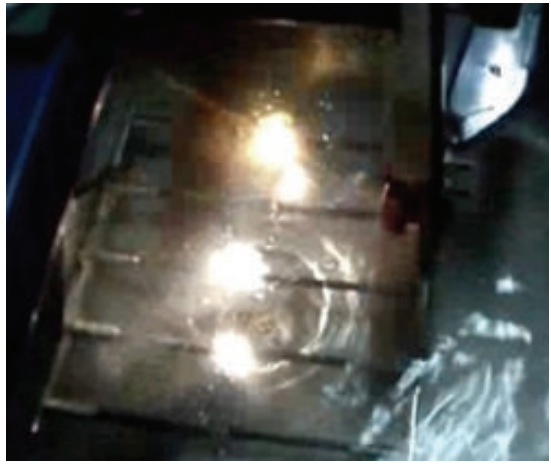

(f)

Figure 4: Continued. 


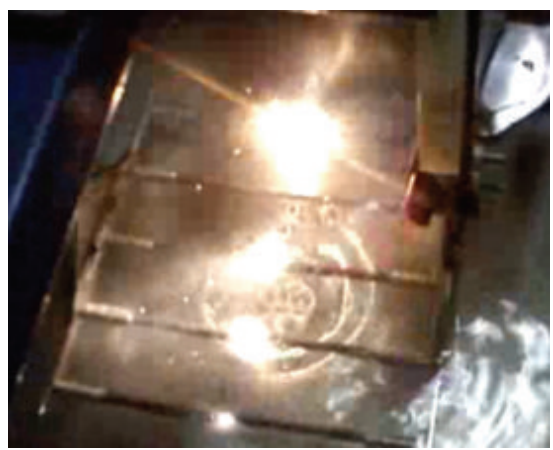

(g)

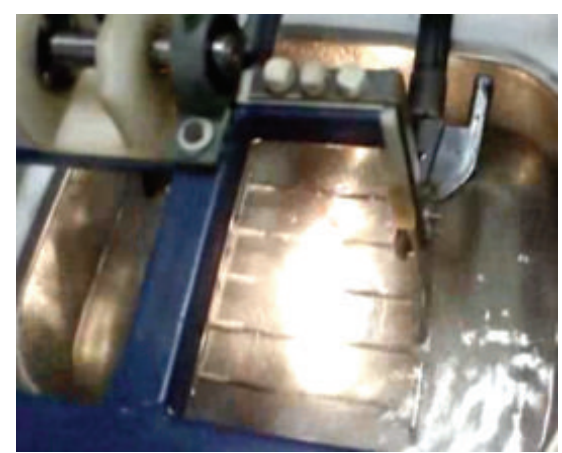

(h)

Figure 4: Clamping the electrodes in fixed and movable holders and the bright plasma region between the multi plasma electrodes; (a) clamping the electrodes, (b) gap sett, (c) initial plasmas, (d) Bright plasmas, (e) two bright plasma, (f) three bright plasma, (g) four bright plasma and (h) formed a fully bright plasma.

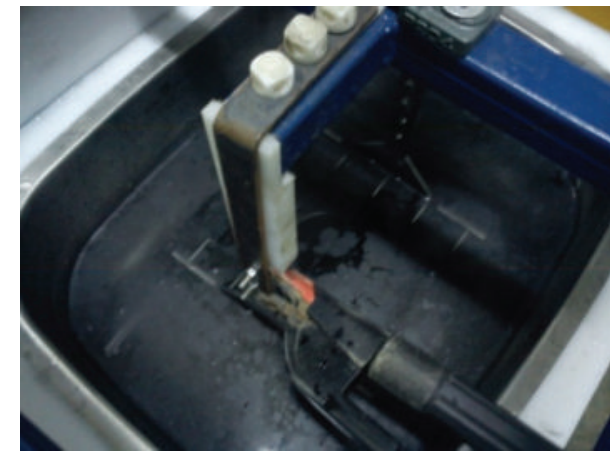

(a)

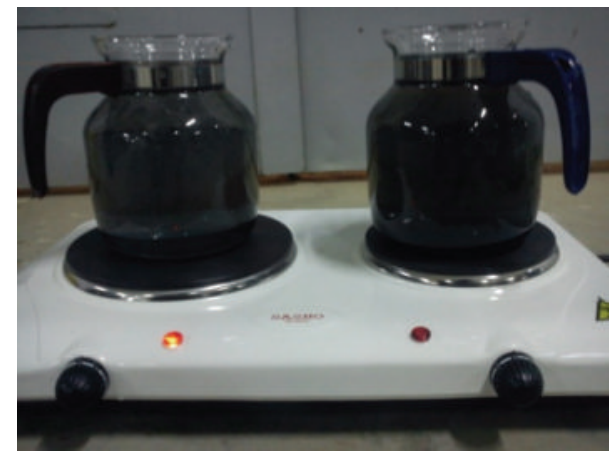

(b)

FIGURE 5: (a) Formed nanoparticles; (b) evaporation of the distilled water.

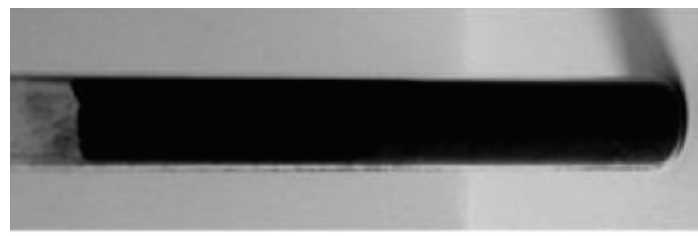

(a)

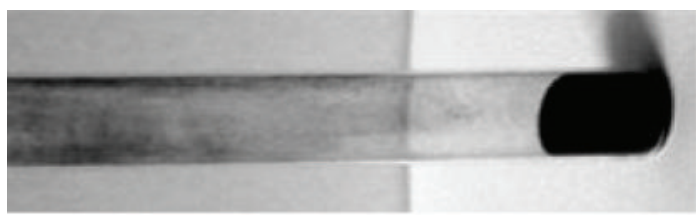

(b)

FIGURE 6: Fully dry soot at (a) multiplasma electrodes and (b) single plasma electrodes.

2.2. Synthesizing MWCNT Using Multiplasma Electrodes. Ten electrodes are used in this experiment. Clamping the ten pure graphite electrodes, five electrodes as cathodes, and five as anodes in the fixed and movable holders, respectively, as shown in Figure 4(a), the experiment is performed under

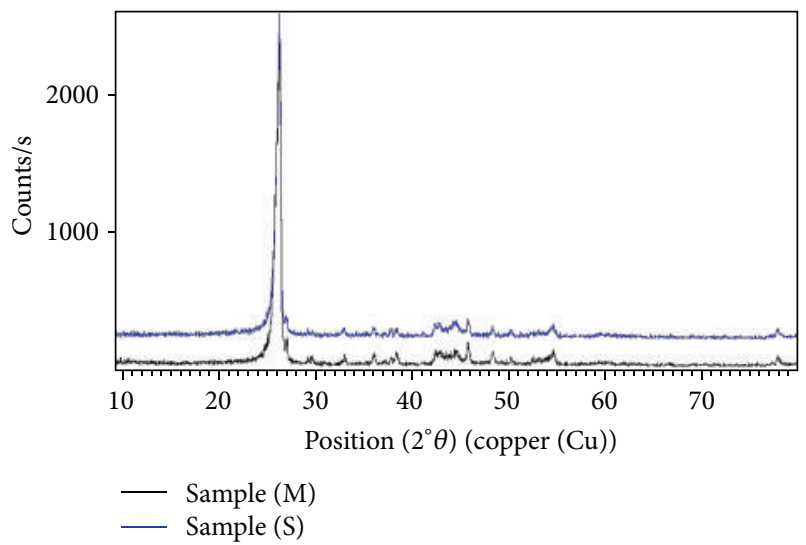

FIgURE 7: XRD pattern of MWNT at single and multi plasma electrodes.

the conditions of AC arc discharge, $75 \mathrm{~A}$ and $238 \mathrm{~V}$, for all electrodes. The arc discharge in this case and the resulted plasma are shown in Figures 4(b)-4(h), respectively. Table 2 summarizes the processing parameters used in this case. In order to reach the strong bright plasma in the multielectrodes arc discharge technique, the electrodes must be submerged to 

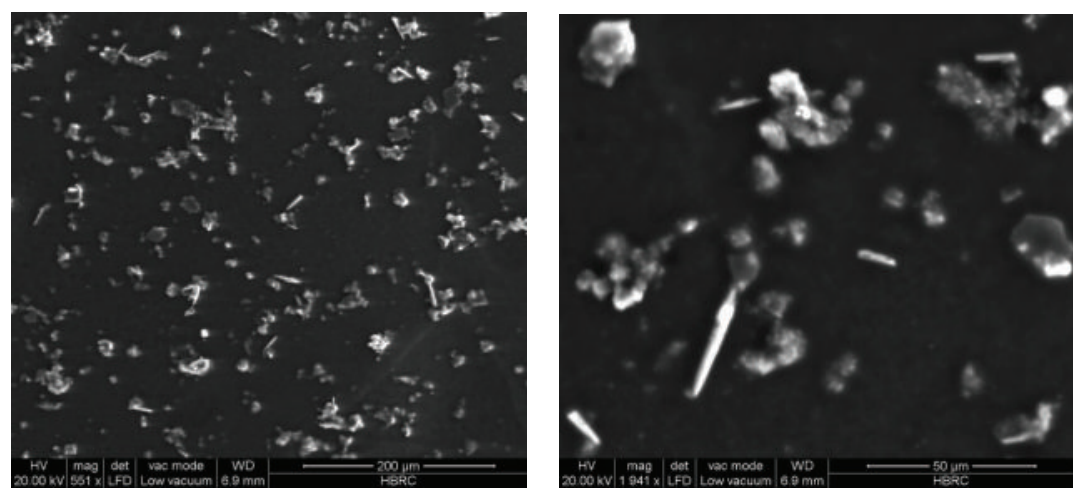

(a) SEM micrograph of MWNT at single plasma electrode case
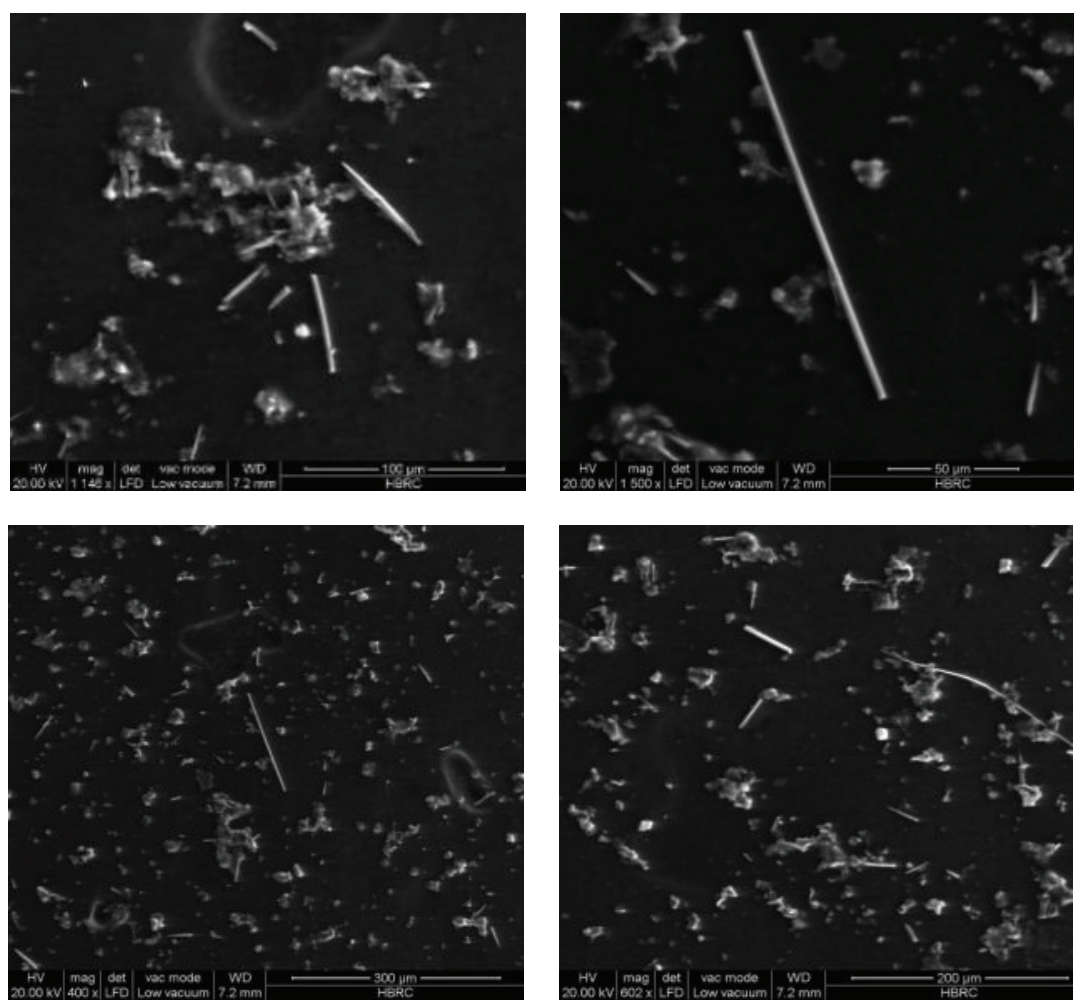

(b) SEM micrograph of MWNT at multiplasma electrodes case

FIGURE 8: SEM images of a synthesized CNT for the studied cases.

a depth of $30 \mathrm{~mm}$ in deionized water. The fixed gap between the faced electrodes is $1 \mathrm{~mm}$. After clamping the multi electrodes in the two holders, the gap between the electrodes ends is set to a distance of $1 \mathrm{~mm}$ from the control unit. Due to the holders assembly manufacturing and electrode dimensions and sizes in terms of tolerances and allowances and probability of small misalignment of electrodes centre, the gaps between all electrodes are not equal. Therefore, the bright plasma generated will not complete until the gaps between all electrodes reach exactly $1 \mathrm{~mm}$. Timer was used to give a signal to the steeper motor every interval of 70 seconds to compensate the electrodes wear.

\section{Sample Preparation}

The generated nano particles can be divided into two types: carbon nano particles that float on the water surface and sediment products, as shown in Figure 5(a). Due to the use of a large quantity of deionized water (about 13 liters) in each experiment, it was found that it is difficult to separate CNT from the deionized water by direct centrifugal. Therefore, it is decided to evaporate the water initially by heating until obtaining about 0.25 liters as shown in Figure 5(b), then to separate CNT by the centrifugal effect. A centrifugal separation device designated L-530 is found sufficient to concentrate the MWCNT. The purification is achieved by successive 


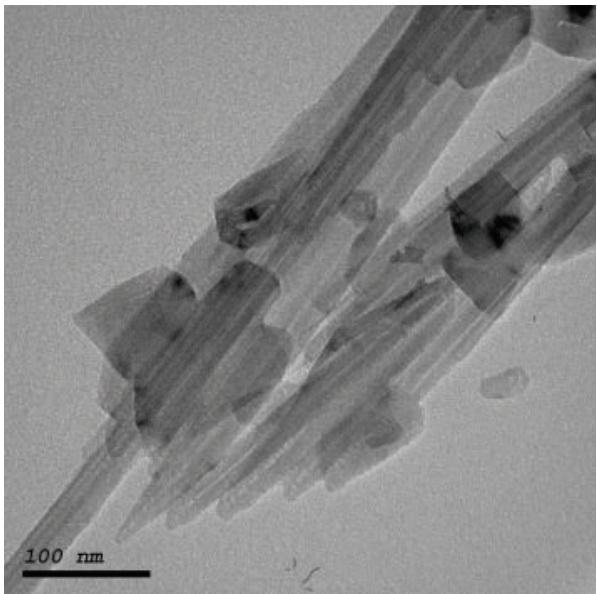

(a)

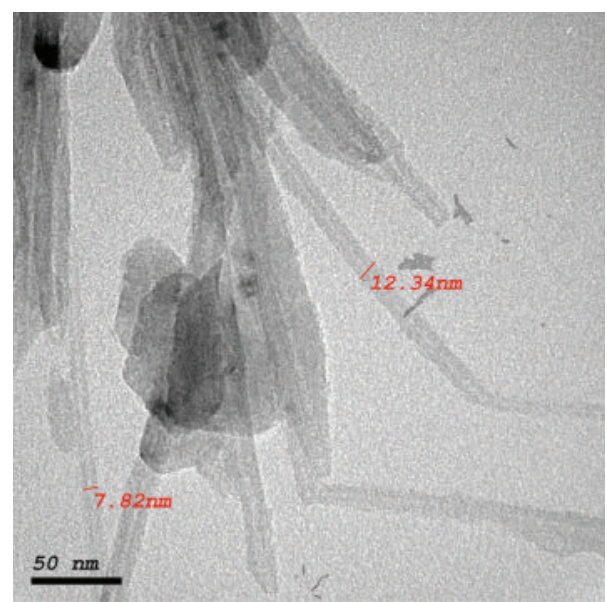

(b)

FIGURE 9: HRTEM image of single plasma electrodes case.

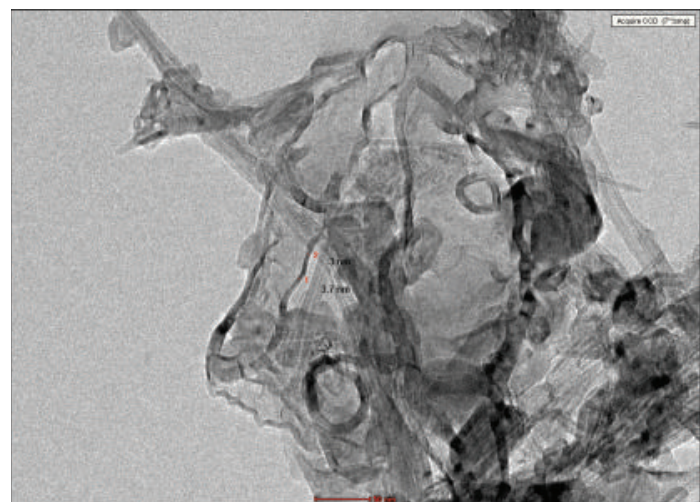

FIGURE 10: HRTEM image of multiplasma electrodes case before oxidation.

separation and decantation by using the centrifugal effect. CNT crude with distilled water was put in the glass tubes in the apparatus with running speed of $4000 \mathrm{rpm}$ for 8 minutes. Due to the high specific gravity, the particles precipitated in the bottom of the glass tubes. After getting rid of the water, the precipitated particles were grouped in one glass tube. The tube was then placed in a furnace at a suitable temperature for fully soot drying as to shown in Figure 6. To find the yield of CNT, soot was heated in a close furnace up to $600^{\circ} \mathrm{C}$ for 2 hours [34-36] then weighed, the soot was weighted before and after oxidation and the percentage weight loss was calculated.

\section{Results and Discussion}

The morphology of MWCNT synthesized by a new design and the degree of purification were observed by SEM BPI-T as well as HRTEM: JEM-2100 and X-ray diffraction (XRD) and Shimadzu TGA-50H for the two different cases; single and multiplasma electrodes were evaluated.
TABLE 2: Experimental conditions for multiplasma electrodes case.

\begin{tabular}{ll}
\hline Parameters & Multiplasma electrodes \\
\hline $\begin{array}{l}\text { Number of } \\
\text { cathodes }\end{array}$ & 5 Graphite Electrodes with $\varnothing 6 \mathrm{~mm}$ \\
$\begin{array}{l}\text { Number of anodes } \\
\text { Applied voltage }\end{array}$ & 5 Graphite Electrodes with $\varnothing 6 \mathrm{~mm}$ \\
$\begin{array}{l}\text { Electric current } \\
\text { Environment }\end{array}$ & $\begin{array}{l}\text { Cooling air, deionized water, and room } \\
\text { temp. }\end{array}$ \\
\hline
\end{tabular}

4.1. X-Ray Diffraction. X-ray diffraction (XRD) is known to be the best method for characterization of CNT structures. Figure 7 shows the XRD pattern of the purified CNT for the two studied cases. At the single plasma electrodes case the strong and sharp reflection peak was found at $26.388^{\circ}$ and for the case of multi electrode at $26.398^{\circ}$. The presence of these peaks in the XRD pattern of CNT indicates the concentric cylindrical nature of graphene sheets nested together and the nanotubes are multi-walled in nature [37].

4.2. Scanning Electron Microscope. Figure 8 illustrates the SEM images of a synthesized CNT at single and multi plasma electrodes. It is clear from the figure that aligned needles tubes resembling spaghetti shape were produced. It is worth noting that the percentages of aligned needle at multi electrodes are much larger than single plasma electrodes. For the purity of MWCNTs, by quantifying the percentage of unwanted materials per unit area within the sample SEM images, it is possible to estimate a degree of purity (e.g., $90 \%$ tubular material, 5\% spherical particles, and 5\% irregular objects) [38], the results have indicated that about 55\% (single plasma case) and 70\% (multi plasma case) were obtained as shown in Figures 8(a)-8(b), respectively. 


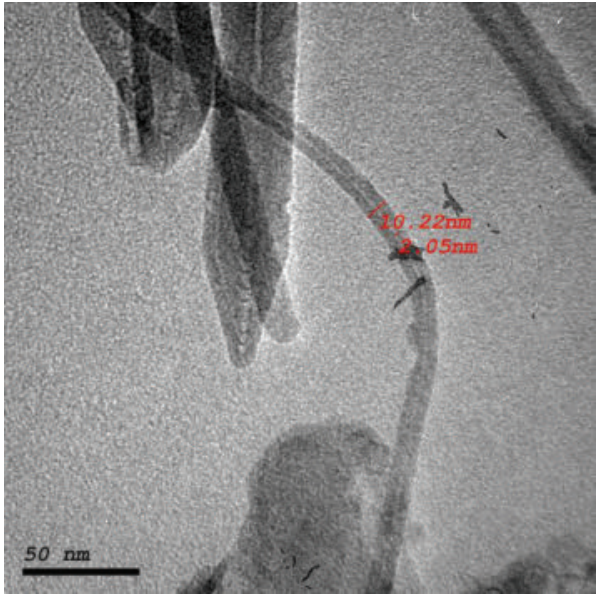

(a)

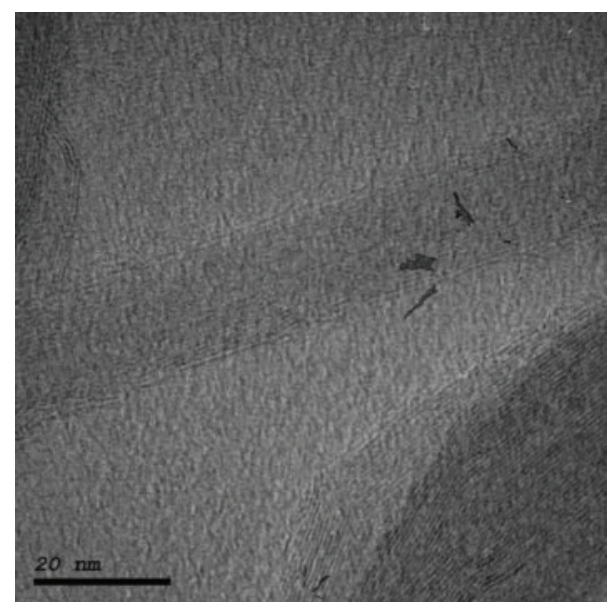

(b)

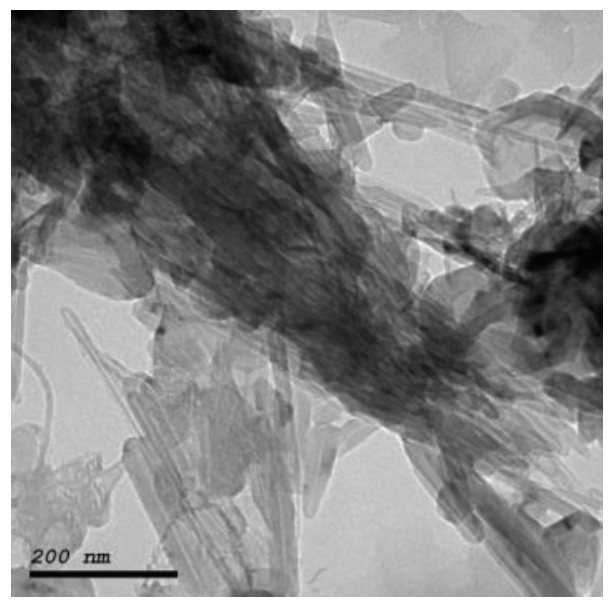

(c)

FIGURE 11: HRTEM image at multi plasma electrodes case after oxidation.

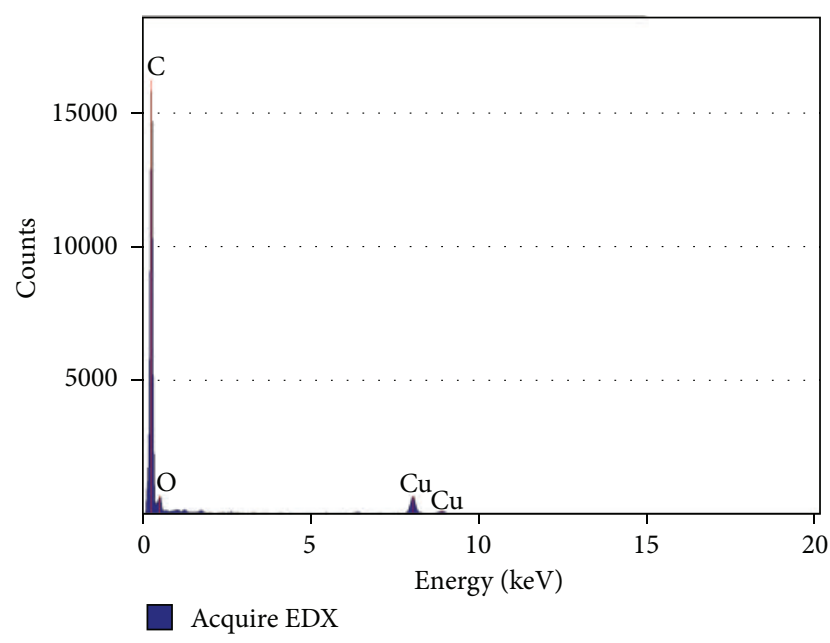

FIGURE 12: EDX analysis shows the high purity carbon.

4.3. High-Resolution Transmission Electron Microscope. HRTEM, JEM-2100, operating at $200 \mathrm{kV}$ was used to characterize the MWNT synthesized for the two studied cases.
At single plasma electrodes case, the TEM images have demonstrated that the resulted was typically of 7-20 nm in diameter, as shown in Figure 9. On the other hand, for the multi plasma electrode CNTs were typically of 3-10 nm in diameter with configuration of nanotubes ends (capped) as shown in Figures 10 and 11. The inset shows the elemental analysis (EDX) which reveal that the total raw samples synthesized for the investigated cases were of highly purity and of good crystallinity. This refers to that there were no other elements found in the analysis, except some minute copper element which resulted from the copper gird in TEM device, as shown in Figure 12.

4.4. Thermogravimetric Analysis. The thermal stability of the synthesized CNT on all samples was analyzed by the thermo gravimetric analysis (TGA). Figure 13 shows the result of the TGA for the studied cases. There is no difference in the diminishing temperature for CNT which were synthesized at single plasma electrodes and multi-plasma electrodes. The graphitic particles started to oxidize at high temperature of about $600^{\circ} \mathrm{C}$. On the other hand, the weight loss in the single 


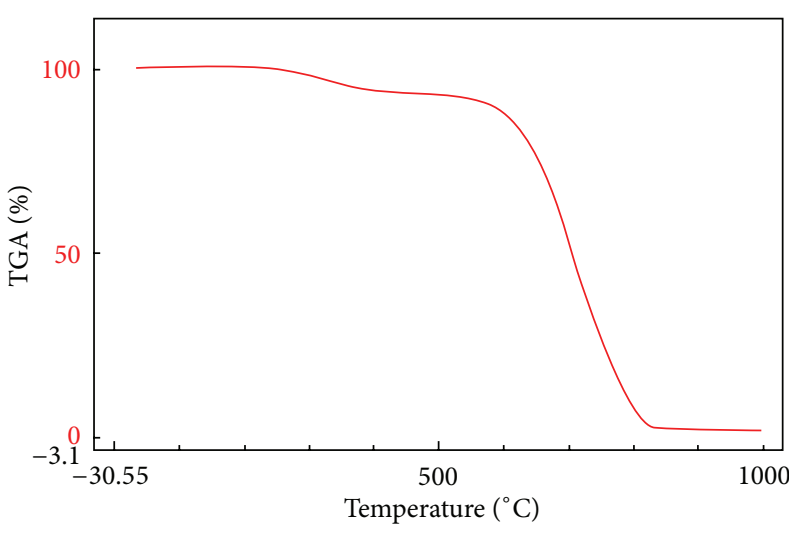

(a)

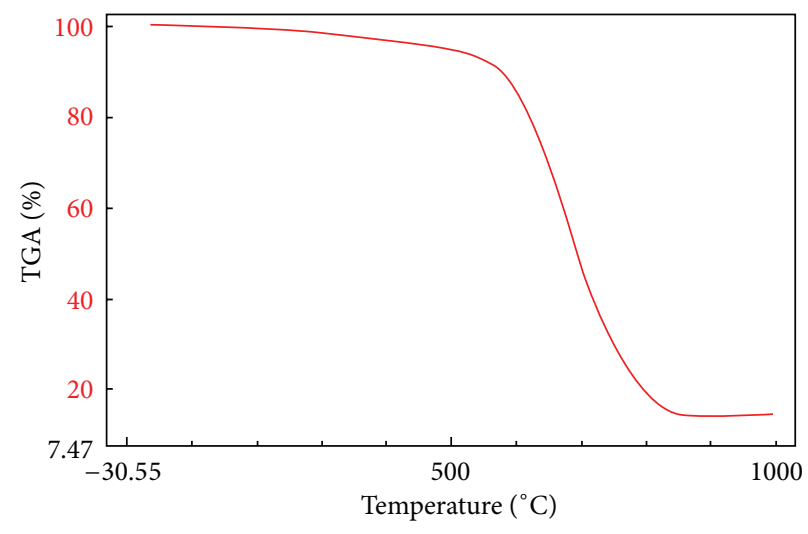

(b)

FIGURE 13: Result of the TGA for (a) single plasma electrodes and (b) multi plasma electrodes.

plasma electrodes is found to be $17 \%$ higher in the case of multi plasma electrodes. Thus, the thermal resistance for the multi electrodes was better than that of the single plasma electrodes.

\section{Conclusion}

The fabrication of MWCNT was achieved by a new designed "fully automatic system" for producing it by using AC arc discharge technique in deionized water for two different cases, the first is single plasma and the second is multi plasma electrodes. The experimental conditions of tests were: $75 \mathrm{~A}$ and $238 \mathrm{~V}$ which produce CNT of $5 \mathrm{~nm}$ in diameter. The total yield in this research was observed to be dependent on the number of the electrodes used in the experiment. The yield of MWCNT in the case of multi electrodes is found to be $320 \%$ higher than that of single plasma electrodes before oxidation. After oxidation, the ratio was found to be nearly the same. Furthermore, it was found that the purity of MWCNT for single plasma electrodes was $40 \%$ yielding with $0.6 \mathrm{~g} / \mathrm{hr}$. On the other hand, the purity of MWCNT for multi plasma electrodes was found to be more than $70 \%$ yielding with $2 \mathrm{~g} / \mathrm{hr}$.

\section{References}

[1] S. Ijima, "Helical microtubules of graphitic carbon," Nature, vol. 354, pp. 56-58, 1991.

[2] H. Shimoda, L. Fleming, K. Horton, and O. Zhou, "Formation of macroscopically ordered carbon nanotube membranes by selfassembly," Physica B, vol. 323, no. 1-4, pp. 135-136, 2002.

[3] P. G. Collins and P. Avouris, "Chapter 3 the electronic properties of carbon nanotubes," Contemporary Concepts of Condensed Matter Science, vol. 3, pp. 49-81, 2008.

[4] H. Rejashree, Y. Manohr, G. Harshal, V. Mohit, and K. Vilasro, "Carbon nanotubes and its applications," Pharmaceutical and Clinical Research, vol. 2, pp. 0974-2441, 2009.

[5] S. Chai, S. Zein, and A. Mohamed, A Review on Carbon Nanotubes Production Via Catalytic Methane Decomposition, NAPCOl, 2004.
[6] J. Prasek, J. Drbohlavova, J. Chomoucka et al., "Methods for carbon nanotubes synthesis-review," Journal of Materials Chemistry, vol. 21, no. 40, pp. 15872-15884, 2011.

[7] C. Journet, W. K. Maser, P. Bernier et al., "Large-scale production of single-walled carbon nanotubes by the electric-arc technique," Nature, vol. 388, no. 6644, pp. 756-758, 1997.

[8] T. Sugai, H. Omote, S. Bandow, N. Tanaka, and H. Shinohara, "Production of fullerenes and single-wall carbon nanotubes by high-temperature pulsed arc discharge," Journal of Chemical Physics, vol. 112, no. 13, pp. 6000-6005, 2000.

[9] Y. Liu, S. Xiaolong, Z. Tingkai, Z. Jiewu, M. Hirscher, and F. Philipp, "Amorphous carbon nanotubes produced by a temperature controlled DC arc discharge," Carbon, vol. 42, no. 8-9, pp. 1852-1855, 2004.

[10] T. Zhao, Y. Liu, and J. Zhu, "Temperature and catalyst effects on the production of amorphous carbon nanotubes by a modified arc discharge," Carbon, vol. 43, no. 14, pp. 2907-2912, 2005.

[11] M. Cadek, R. Murphy, B. McCarthy et al., "Optimisation of the arc-discharge production of multi-walled carbon nanotubes," Carbon, vol. 40, no. 6, pp. 923-928, 2002.

[12] Y. Liu, S. Xiaolong, Z. Tingkai, Z. Jiewu, M. Hirscher, and F. Philipp, "Amorphous carbon nanotubes produced by a temperature controlled DC arc discharge," Carbon, vol. 42, no. 8-9, pp. 1852-1855, 2004.

[13] H. Qiu, Z. Shi, L. Guan et al., "High-efficient synthesis of double-walled carbon nanotubes by arc discharge method using chloride as a promoter," Carbon, vol. 44, no. 3, pp. 516-521, 2006.

[14] R. B. Mathur, S. Seth, C. Lal et al., "Co-synthesis, purification and characterization of single- and multi-walled carbon nanotubes using the electric arc method," Carbon, vol. 45, no. 1, pp. 132-140, 2007.

[15] S.-D. Wang, M.-H. Chang, J.-J. Cheng, H.-K. Chang, and K. M.-D. Lan, "Unusual morphologies of carbon nanoparticles obtained by arc discharge in deionized water," Carbon, vol. 43, no. 6, pp. 1322-1325, 2005.

[16] A. Qader, A. S. Keiteb, and M. A. Jaleel, "Synthesis of carbon nanomaterials in deionized water with and without catalyst using arc discharge technique," Engineering Technician, vol. 29, no. 2, 2011.

[17] A. Aqel, K. M. M. A. El-Nour, R. A. A. Ammar, and A. AlWarthan, "Carbon nanotubes, science and technology part (I) structure, synthesis and characterisation," Arabian Journal of Chemistry, vol. 5, no. 1, pp. 1-23, 2012. 
[18] A. Thess, R. Lee, P. Nikolaev et al., "Crystalline ropes of metallic carbon nanotubes," Science, vol. 273, no. 5274, pp. 483-487, 1996.

[19] M. J. Y. Yacaman, M. M. Yoshida, and L. Rendon, "Catalytic growth of carbon microtubules with fullerene structure," Applied Physics Letters, vol. 62, no. 2, pp. 202-204, 1993.

[20] G. Che, B. B. Lakshmi, C. R. Martin, E. R. Fisher, and R. S. Ruoff, "Chemical vapor deposition based synthesis of carbon nanotubes and nanofibers using a template method," Chemistry of Materials, vol. 10, no. 1, pp. 260-267, 1998.

[21] H. W. Zhu, C. L. Xu, D. H. Wu, B. Q. Wei, R. Vajtai, and P. M. Ajayan, "Direct synthesis of long single-walled carbon nanotube strands," Science, vol. 296, no. 5569, pp. 884-886, 2002.

[22] M. Keidar and A. M. Waas, "On the conditions of carbon nanotube growth in the arc discharge," Nanotechnology, vol. 15, no. 11, pp. 1571-1575, 2004.

[23] N. Grobert, “Carbon nanotubes-becoming clean," Materials Today, vol. 10, no. 1-2, pp. 28-35, 2007.

[24] M. Ishigami, J. Cumings, A. Zettl, and S. Chen, "A simple method for the continuous production of carbon nanotubes," Chemical Physics Letters, vol. 319, no. 5-6, pp. 457-459, 2000.

[25] Y. S. Park, K. S. Kim, H. J. Jeong, W. S. Kim, J. M. Moon, K. H. An et al., "Low pressure synthesis of single-walled carbon nanotubes by arc discharge," Synthetic Metals, vol. 126, no. 2-3, pp. 245-251, 2002.

[26] M. Teymourzadeh and H. Kangarlou, "Synthesis of multiwalled carbon nanotubes in an arc discharge using hydrocarbons precursor as carbon sources," Sciences, vol. 18, no. 7, pp. 879-883, 2012.

[27] Z. Shi, Y. Lian, X. Zhou et al., "Mass-production of single-wall carbon nanotubes by arc discharge method," Carbon, vol. 37, no. 9, pp. 1449-1453, 1999.

[28] M. Jahanshahi, M. Shariaty-Niassar, A. A. Rostami, H. Molavi, and F. Toubi, "Arc-discharge carbon nanotube fabrication in solution: electrochemistry and voltametric tests," Australian Journal of Basic and Applied Sciences, vol. 4, no. 12, pp. 59155922, 2010.

[29] H. W. Zhu, X. S. Li, B. Jiang et al., "Formation of carbon nanotubes in water by the electric-arc technique," Chemical Physics Letters, vol. 366, no. 5-6, pp. 664-669, 2002.

[30] M. V. Antisari, R. Marazzi, and R. Krsmanovic, "Synthesis of multiwall carbon nanotubes by electric arc discharge in liquid environments," Carbon, vol. 41, no. 12, pp. 2393-2401, 2003.

[31] S. J. Lee, H. K. Baik, J. E. Yoo, and J. H. Han, "Large scale synthesis of carbon nanotubes by plasma rotating arc discharge technique," Diamond and Related Materials, vol. 11, no. 3-6, pp. 914-917, 2002.

[32] L. P. Biro, Z. E. Horvath, L. Szalmas, K. Kertesz, F. Weber, G. Juhasz et al., "Continuous carbon nanotube production in underwater AC electric arc," Chemical Physics Letters, vol. 372, no. 3-4, pp. 399-402, 2003.

[33] S. Yousef, A. khattab, T. A. Osman, and M. Zaki, Fully Automatic System For Producing Carbon Nanotubes (CNTs) By Using ArcDischarge Technique Multi Electrodes, ICIES, 2012.

[34] P. M. Ajayan, T. W. Ebbesen, T. Ichihashi, S. Ijijima, K. Tanigaki, and H. Hiura, "Opening carbon nanotubes with oxygen and implications for filling," Nature, vol. 362, no. 6420, pp. 522-525, 1993.

[35] M. C. Paladugu, K. Maneesh, P. K. Nair, and P. Haridoss, "Synthesis of carbon nanotubes by arc discharge in open air," Journal of Nanoscience and Nanotechnology, vol. 5, no. 5, pp. 747-752, 2005.
[36] R. Joshi, J. Engstler, P. K. Nair, P. Haridoss, and J. J. Schneider, "High yield formation of carbon nanotubes using a rotating cathode in open air," Diamond and Related Materials, vol. 17, no. 6, pp. 913-919, 2008.

[37] M. S. S. Saravanan, S. P. K. Babu, K. Sivaprasad, and M. Jagannatham, "Technoeconomics of carbon nanotubes produced by open air arc discharge method," Journal of Engineering Science and Technology, vol. 2, no. 5, pp. 100-108, 2010.

[38] J. H. Lehman, M. Terrones, E. Mansfield, K. E. Hurst, and V. Meunier, "Evaluating the characteristics of multiwall carbon nanotubes," Carbon, vol. 49, no. 8, pp. 2581-2602, 2011. 

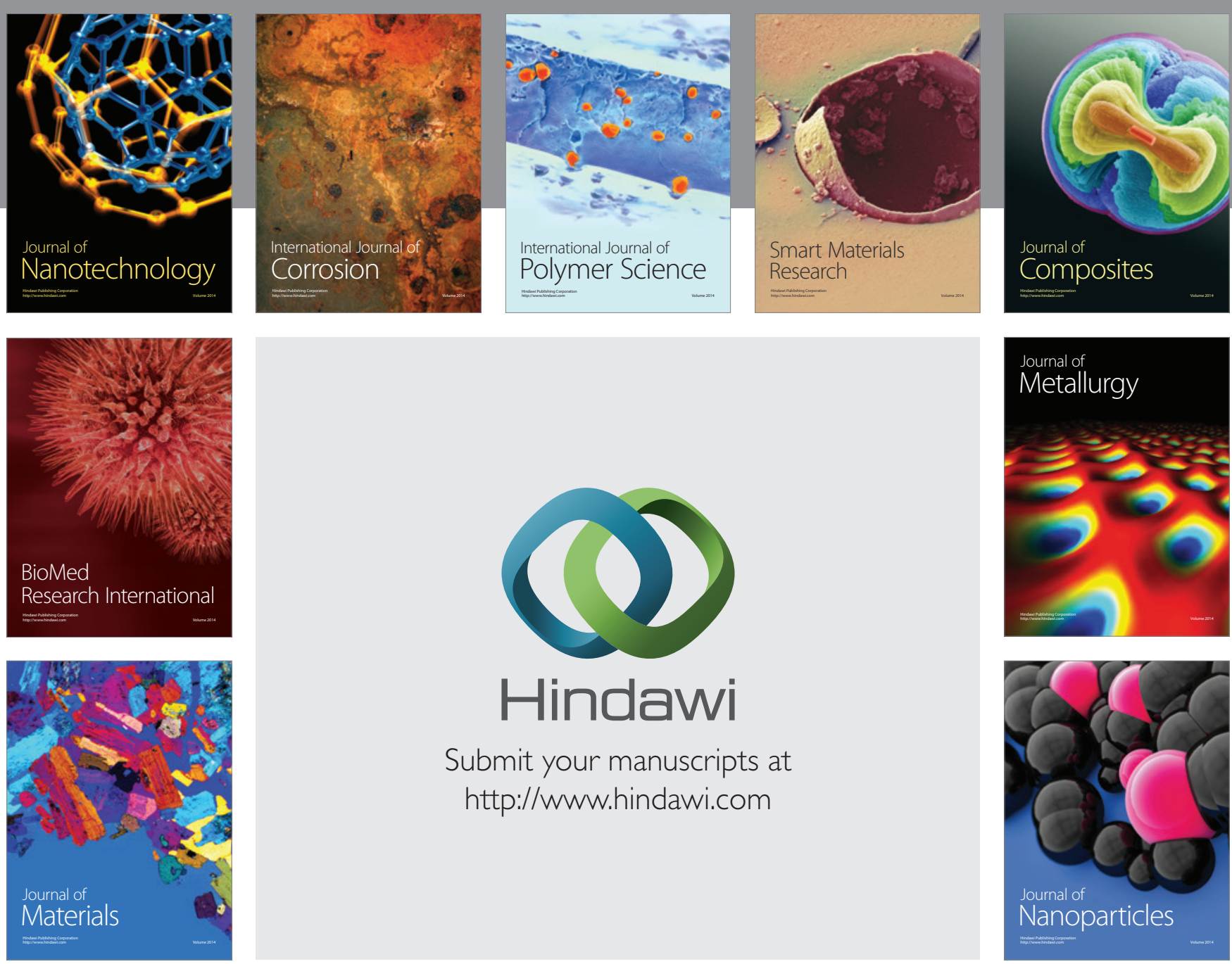

Submit your manuscripts at http://www.hindawi.com
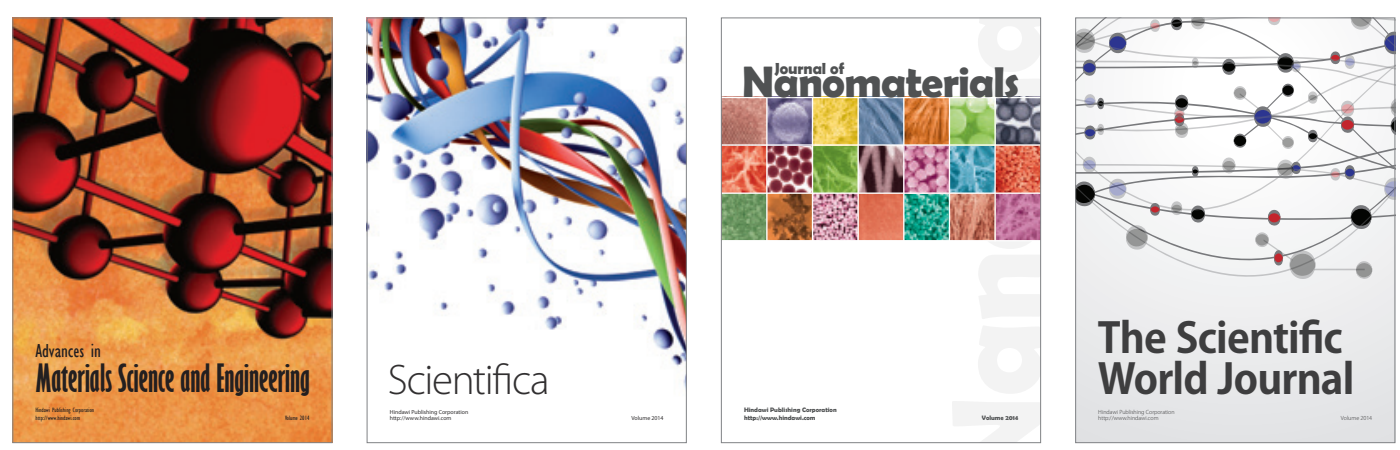

\section{The Scientific World Journal}
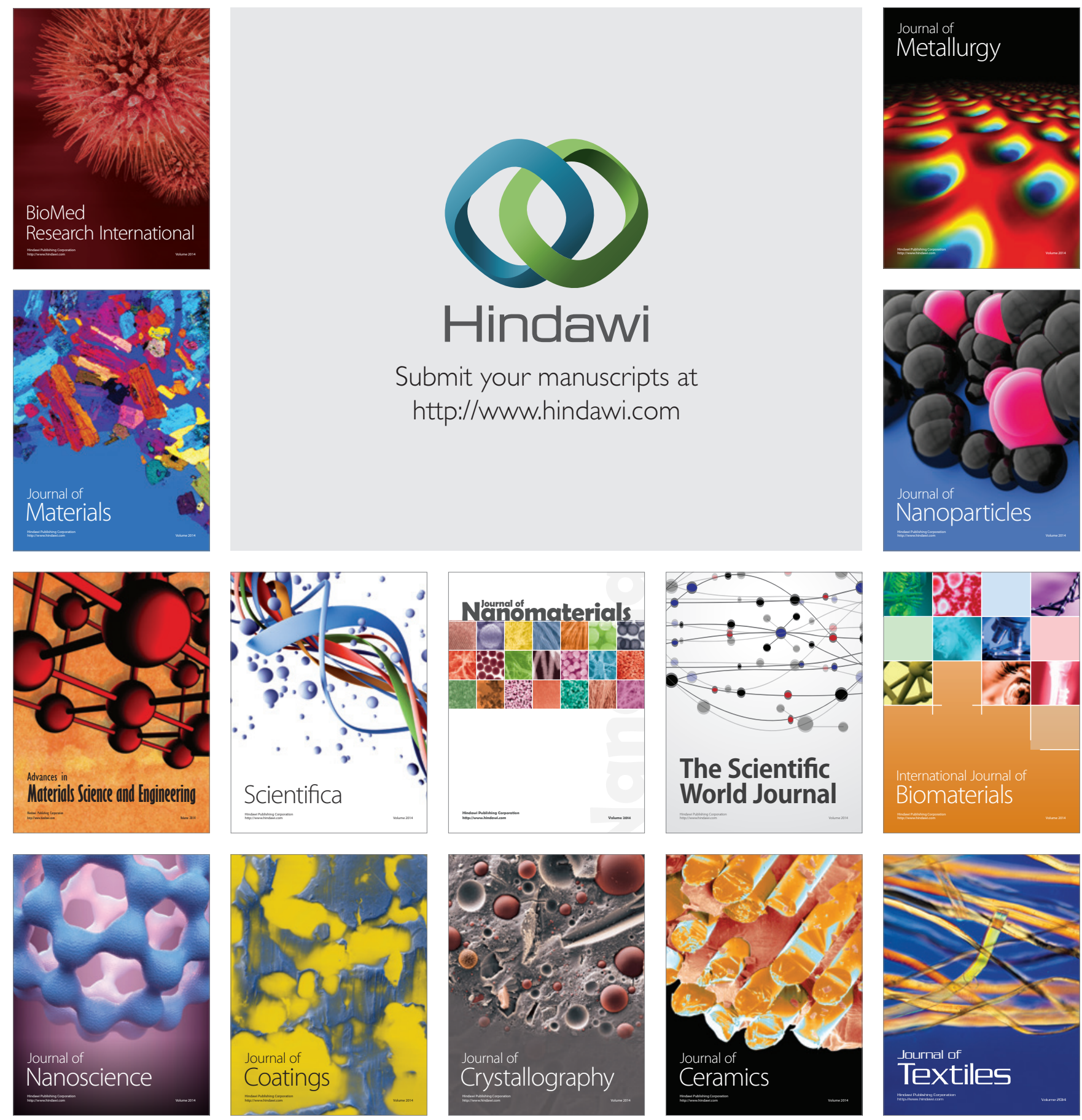